\title{
Promising nitrogen-rich porous carbons derived from one-step calcium chloride activation of biomass-based waste for high-performance supercapacitors
}

\author{
Jingjiang Liu, ${ }^{\dagger}$ Yuanfu Deng, ${ }^{*}{ }^{\dagger}, \dagger$, Xuehui Li, ${ }^{*}, \dagger, \S$ Lefu Wang, ${ }^{\dagger}$ \\ ${ }^{\dagger}$ School of Chemistry and Chemical Engineering, South China University of \\ Technology, Guangzhou, 510640, China; \\ The Key Laboratory of Fuel Cell Technology of Guangdong province; \\ ${ }^{\S}$ Pulp \& Paper Engineering State Key Laboratory of China.
}


SEM images of $\mathrm{S}_{100}$ (Figure $\mathrm{S} 1$ ) and $\mathrm{S}_{102}$ (Figure $\mathrm{S} 2$ ), pore diameter distribution curves of all samples (Figure S3), Nitrogen adsorption-desorption isotherms of the $\mathrm{ZnCl}_{2}-\mathrm{S}_{122}$ (Figure S4) and $\mathrm{KOH}-\mathrm{S}_{122}$ (Figure S5), XRD patterns of the precursor and intermediate products (Figure S6), the magnified region of Ramam spectra (Figure S7), high-resolution XPS of N1s region for $S_{102}$ (Figure S8), $S_{121}$ (Figure S9) and $S_{122}$ (Figure S10), discharge capacitances of all of the obtained electrodes at various current densities (Figure S11) and (Figure S12), the cycle stability of $\mathrm{S}_{122}$ electrode at $5 \mathrm{~A} \mathrm{~g}^{-1}$ (Figure S13), the specific capacitances of $\mathrm{S}_{122}$ at different densities in two-electrode system (Figure S14), the percentage of mesopores of the as-prepared samples (Table $\mathrm{S} 1$ ), approximate distribution of $\mathrm{N}$ functional groups obtained by fitting the $N_{1 s}$ core level XPS spectra of the $S_{122}$ and $S_{102}$ samples (Table $S 2$ ), comparison of the BET surface area, pore volume and capacitance of different $\mathrm{N}$-doped activated carbons in the literatures (Table S3), IR drops of the $\mathrm{S}_{121}$ electrode at different current densities (Table S4) and The EA results, specific surface area and specific capacitance of the as-prepared samples obtained from different temperatures (Table S5). 


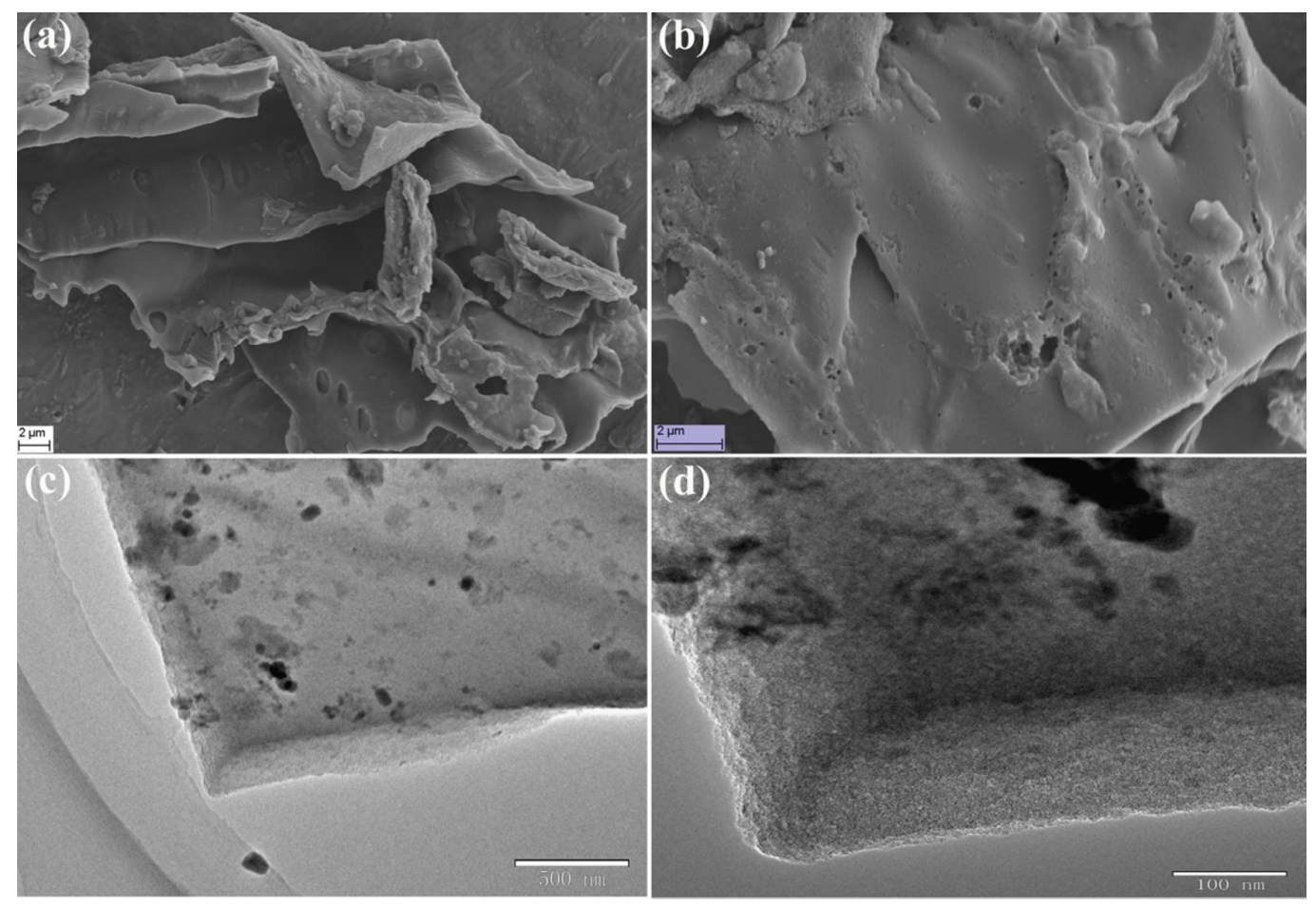

Figure S1. SEM images of the $(a, b) S_{100}$; TEM images of the (c, d) $S_{100}$. 

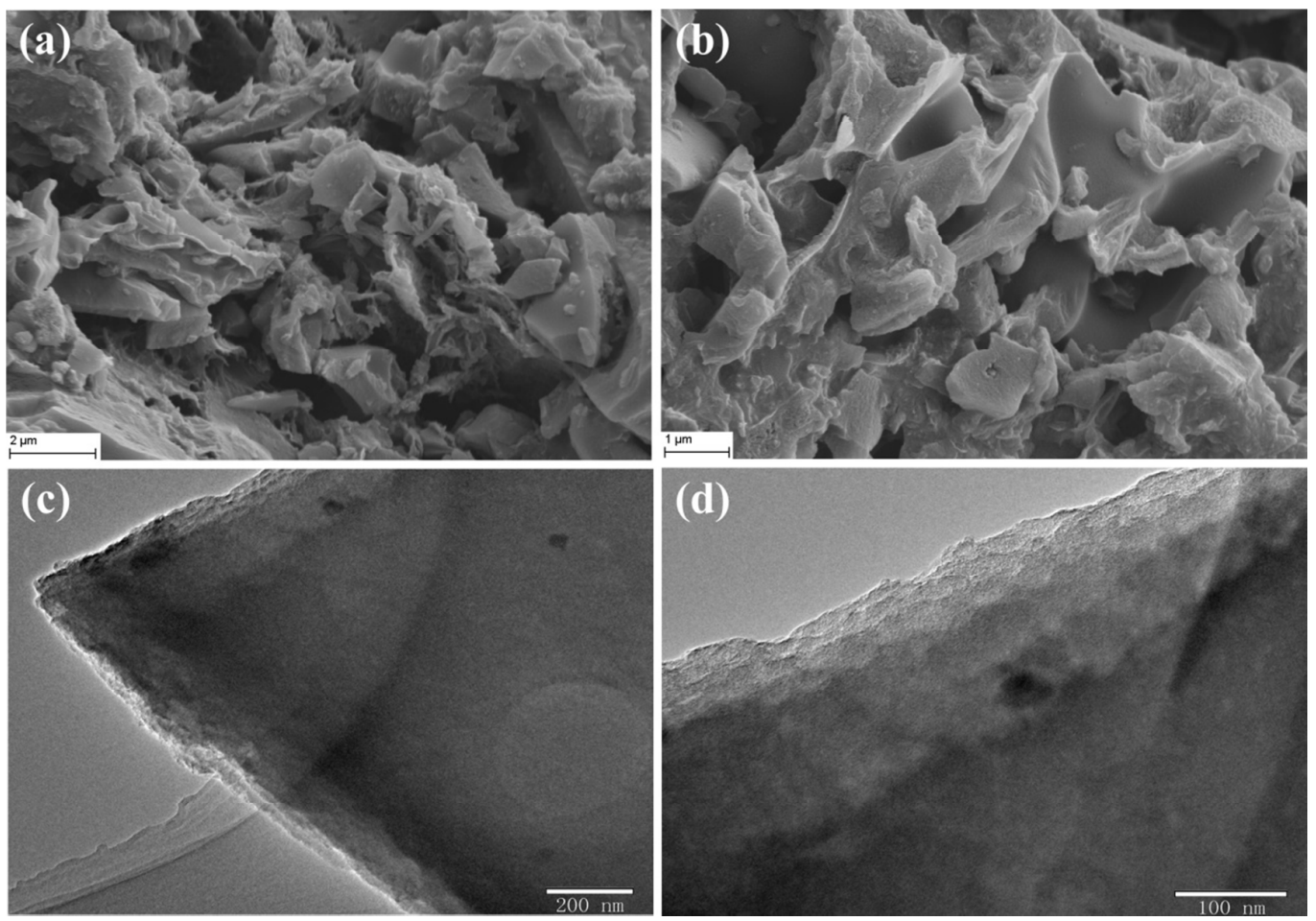

Figure S2. SEM images of the (a, b) $\mathrm{S}_{102}$; TEM images of the (c, d) $\mathrm{S}_{102}$. 


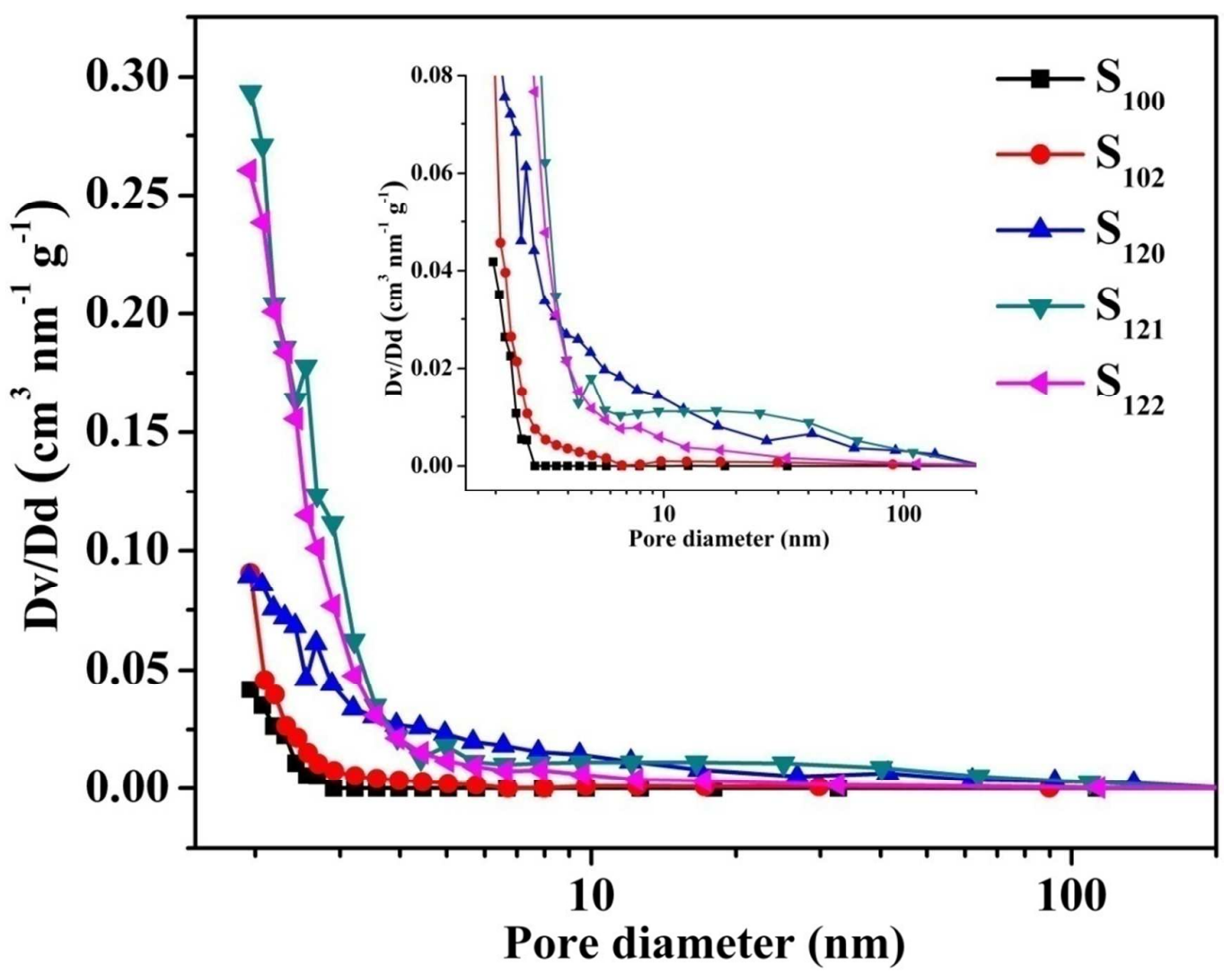

Figure S3. Pore diameter distribution curves of all the obtained samples. 


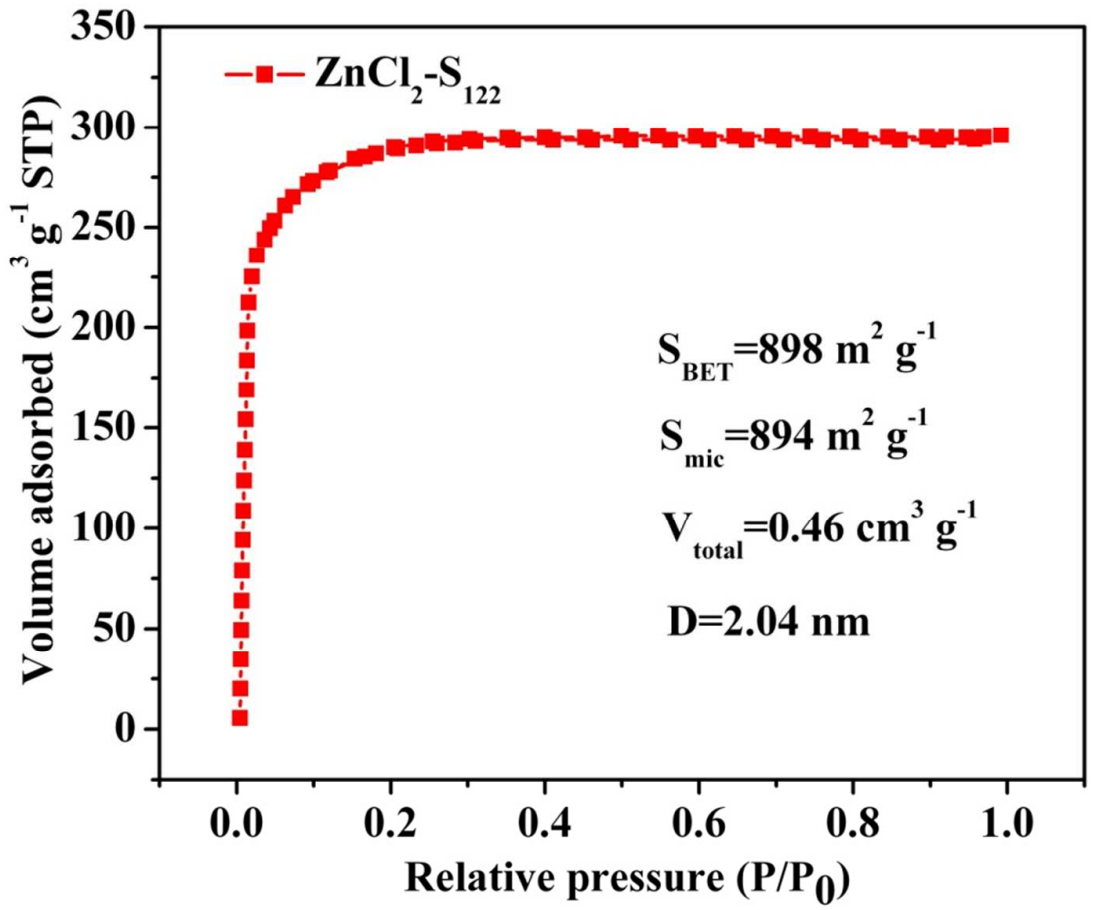

Figure S4. Nitrogen adsorption-desorption isotherms of the $\mathrm{ZnCl}_{2}-\mathrm{S}_{122}$ sample. 


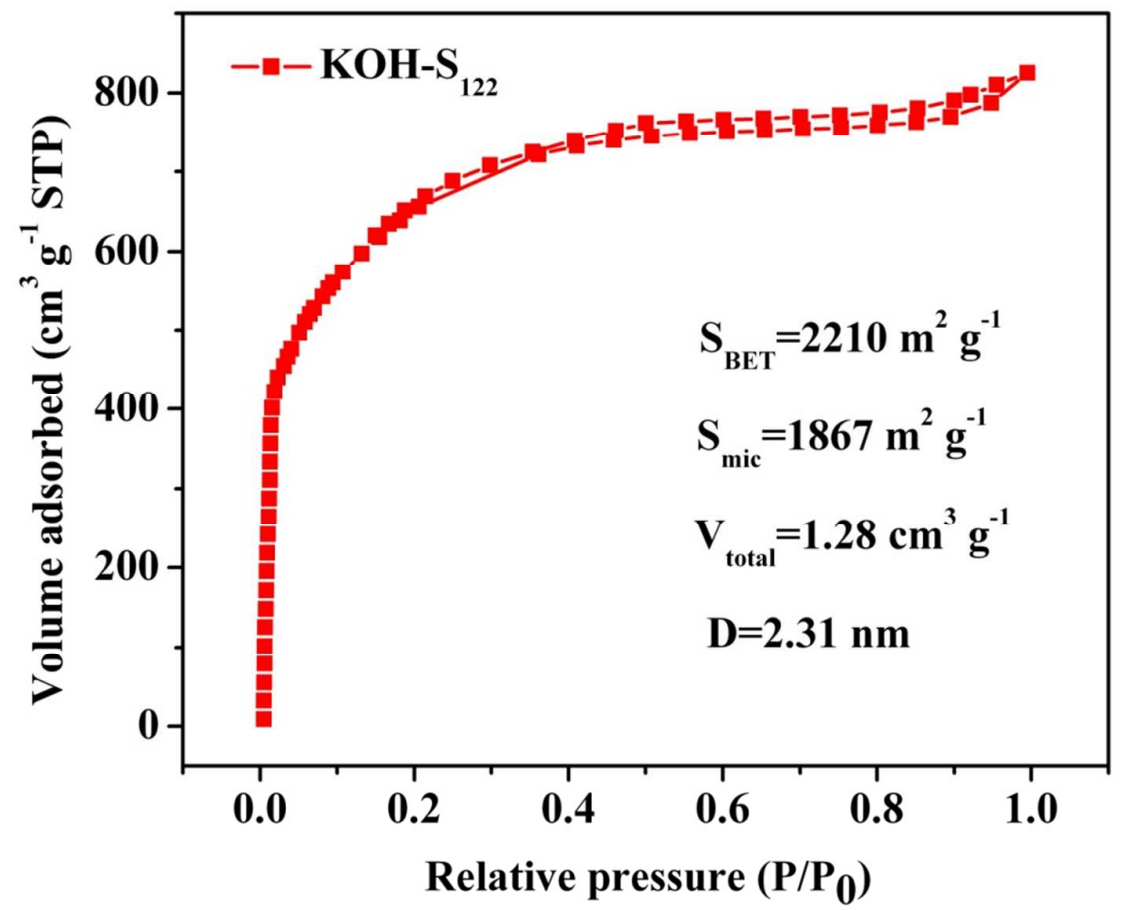

Figure S5. Nitrogen adsorption-desorption isotherms of the KOH-S 122 sample. 


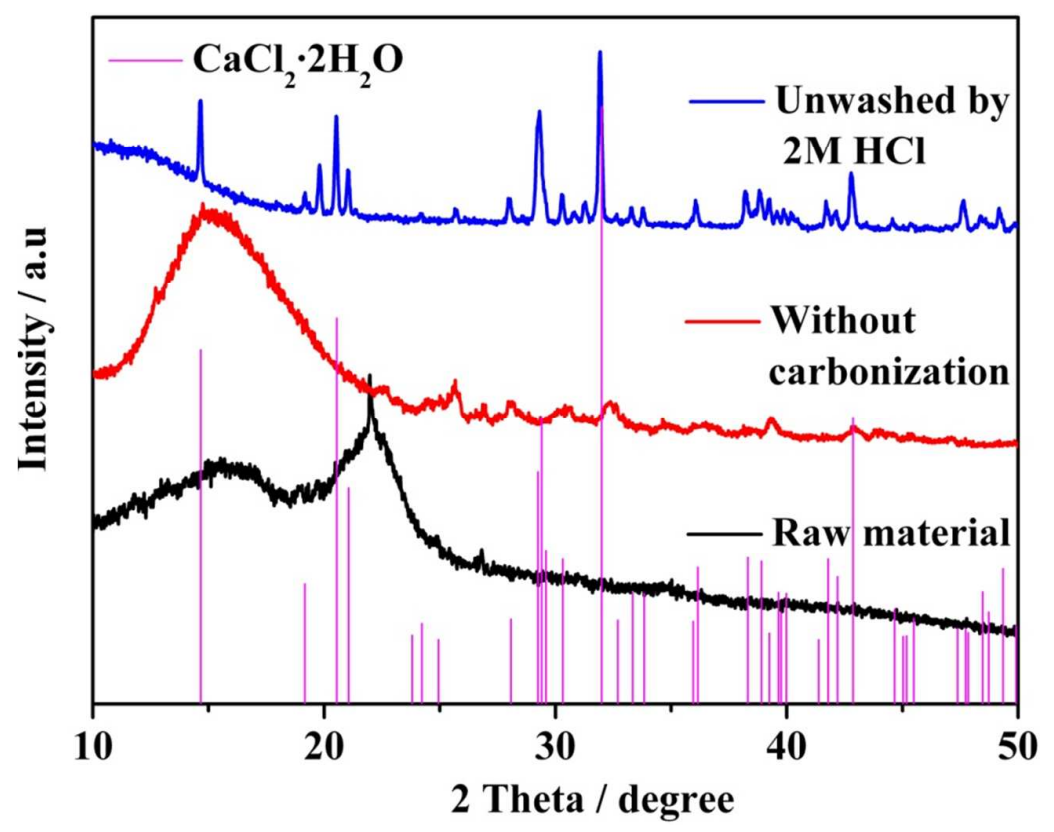

Figure S6. XRD patterns of the precursor and intermediate products. 


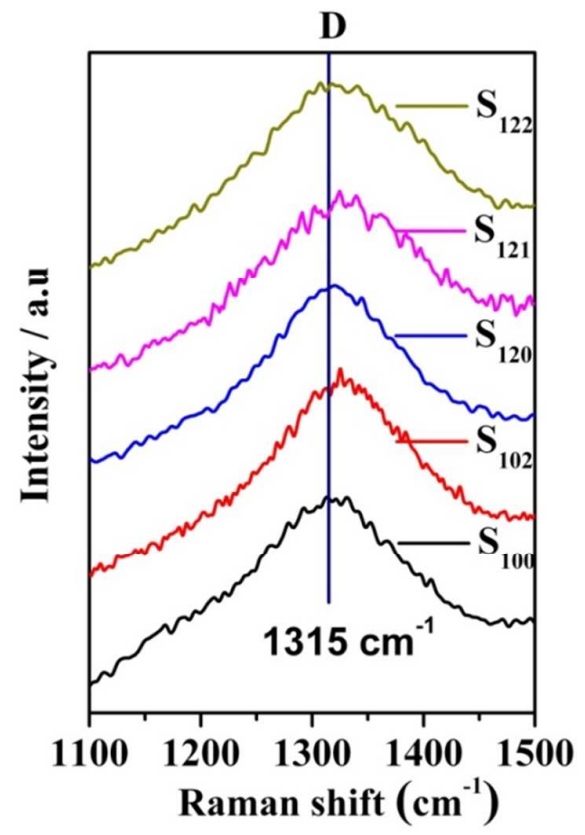

Figure 1. The Raman spectra for the as-prepared samples showing the D peaks. 


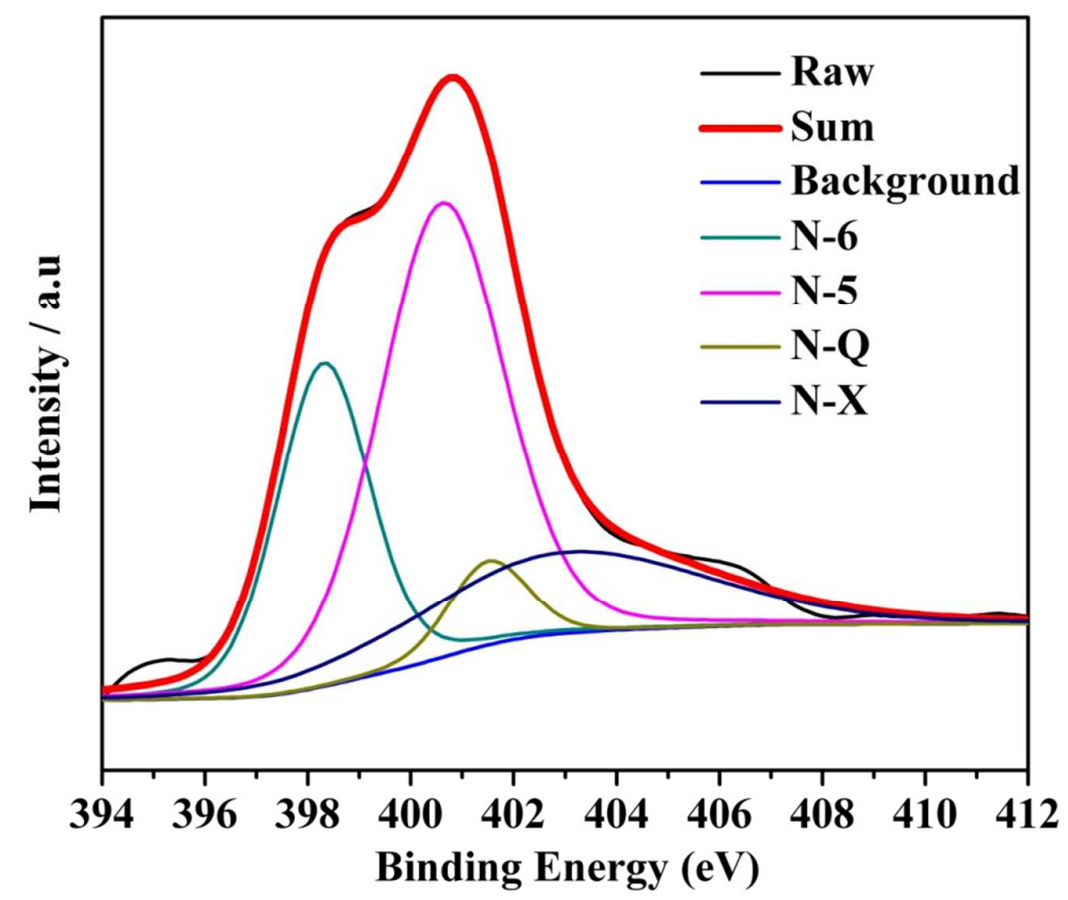

Figure S8. High-resolution X-ray photoelectron spectroscopy (XPS) of N1s region for $\mathrm{S}_{102}$ sample. 


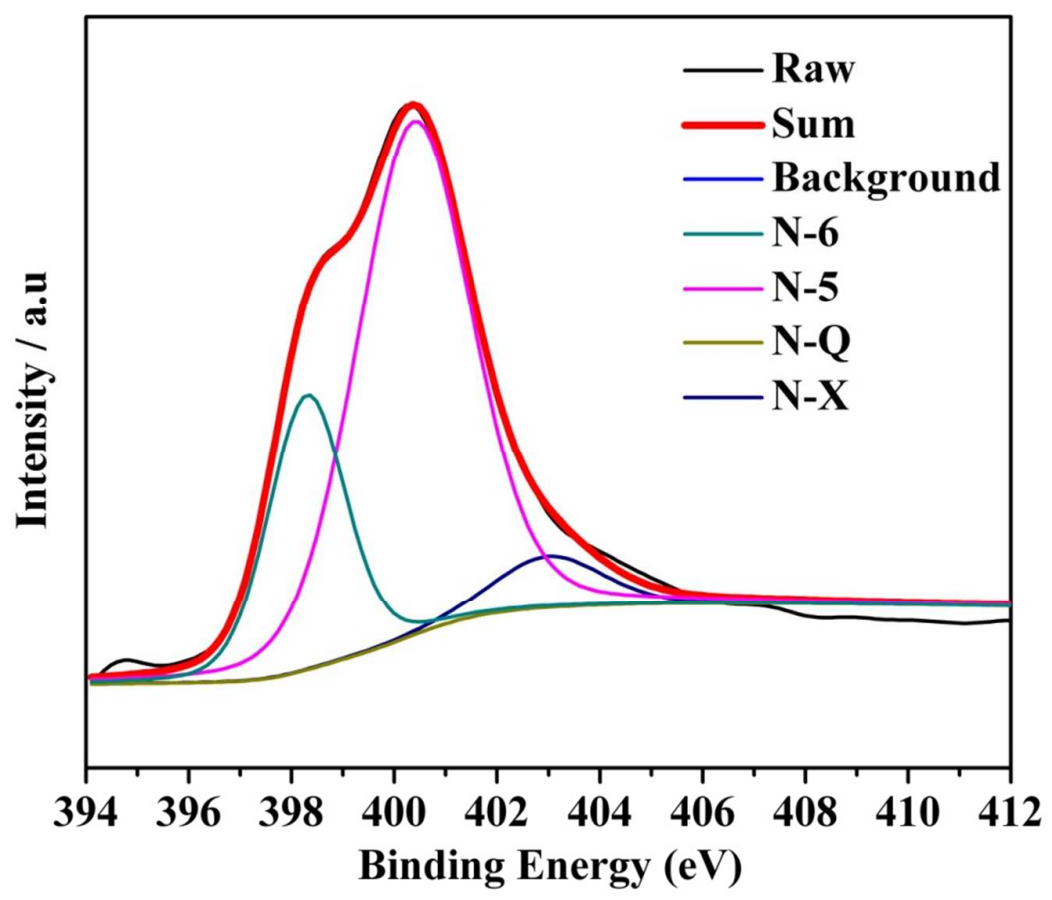

Figure S9. High-resolution X-ray photoelectron spectroscopy (XPS) of N1s region for $S_{121}$ sample. 


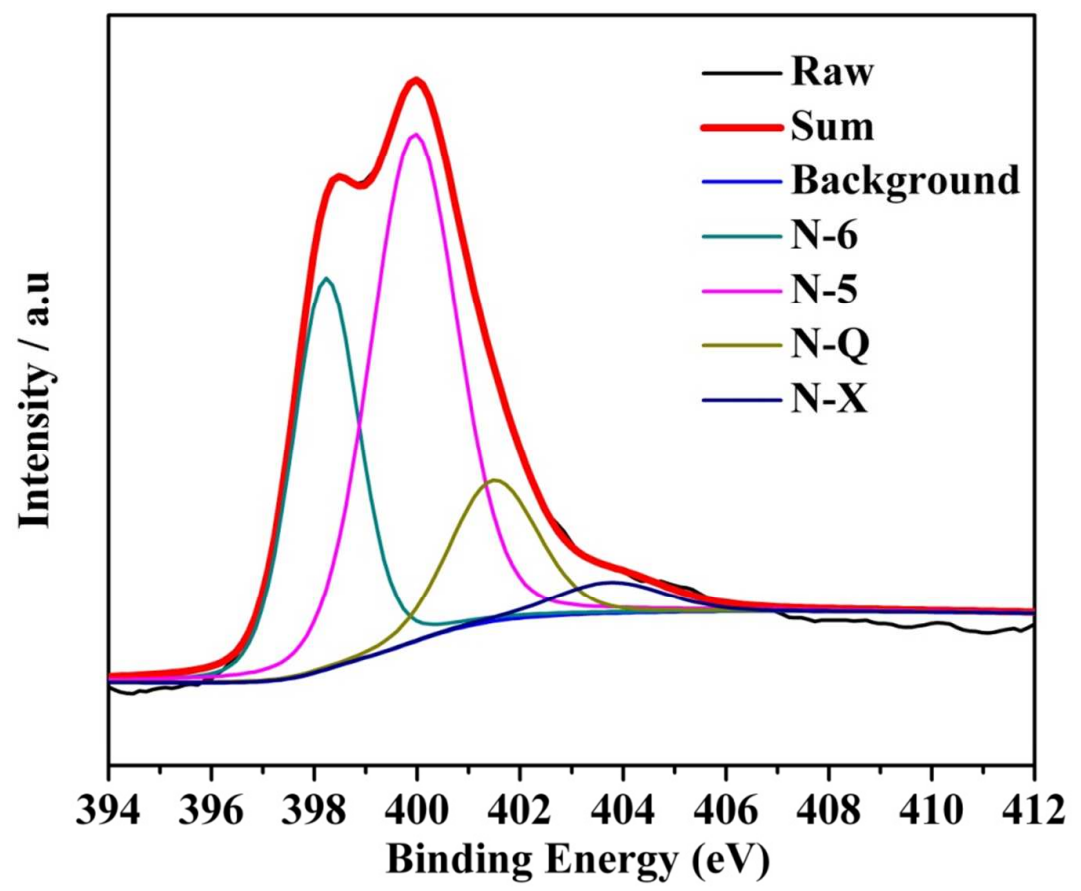

Figure S10. High-resolution X-ray photoelectron spectroscopy (XPS) of N1s region for $S_{122}$ sample. 


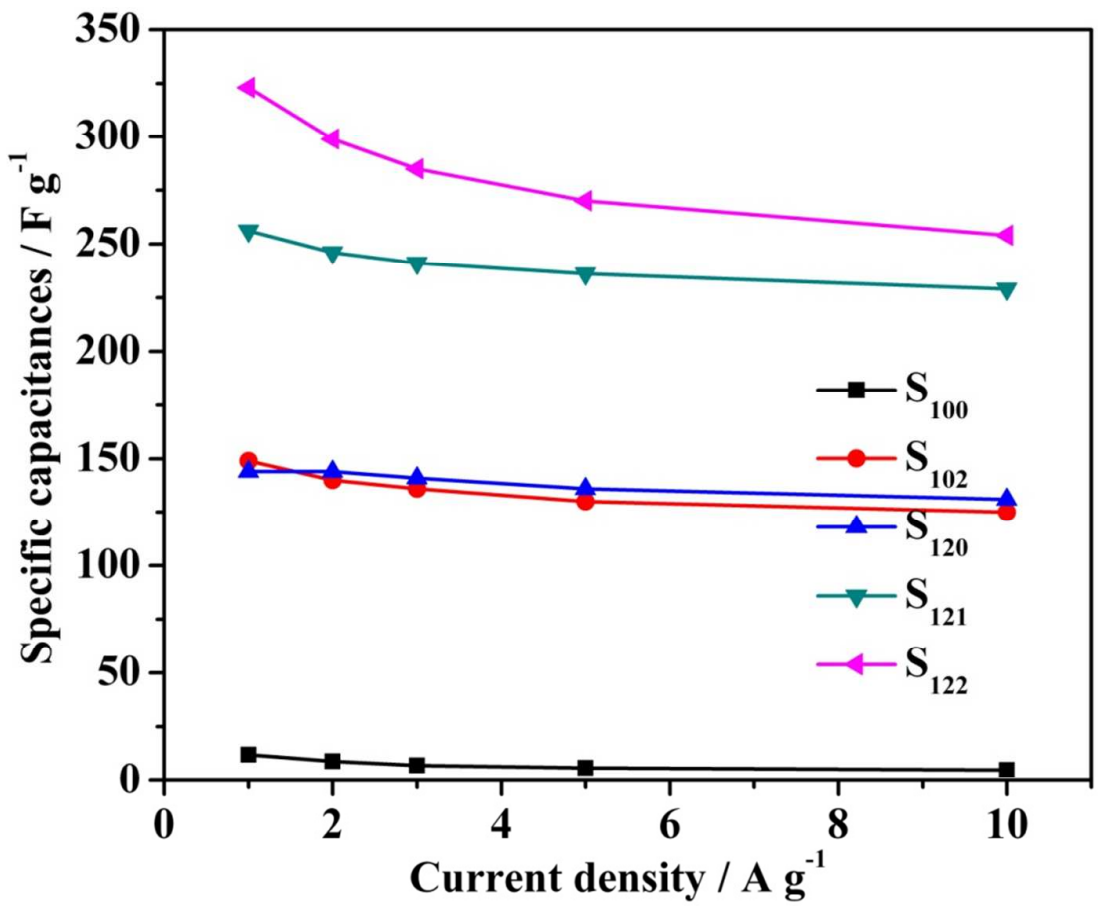

Figure S11. Discharge capacitances of all of the obtained electrodes at various current densities. 


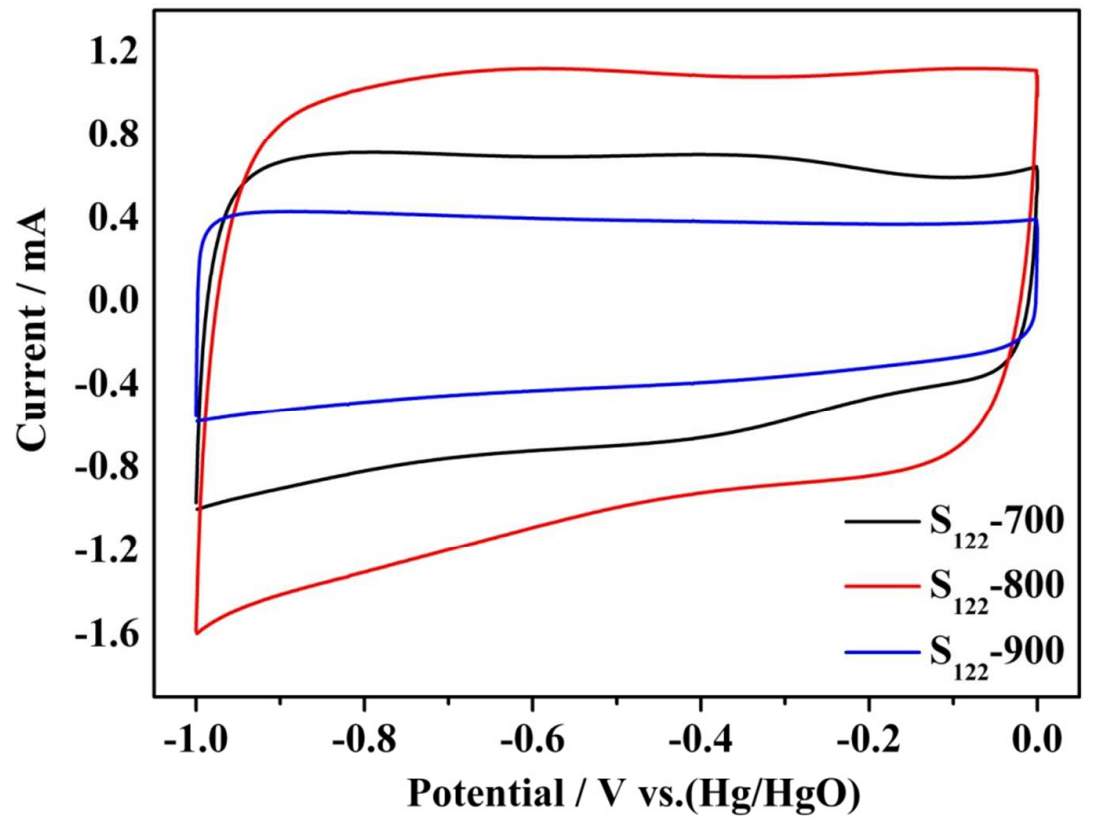

Figure S12. The CV curves of the $S_{122-}-700, S_{122}-800$ and $S_{122}-900$ at $50 \mathrm{mV} \mathrm{s}^{-1}$ in 6.0 $\mathrm{M} \mathrm{KOH}$ aqueous electrolytes. 


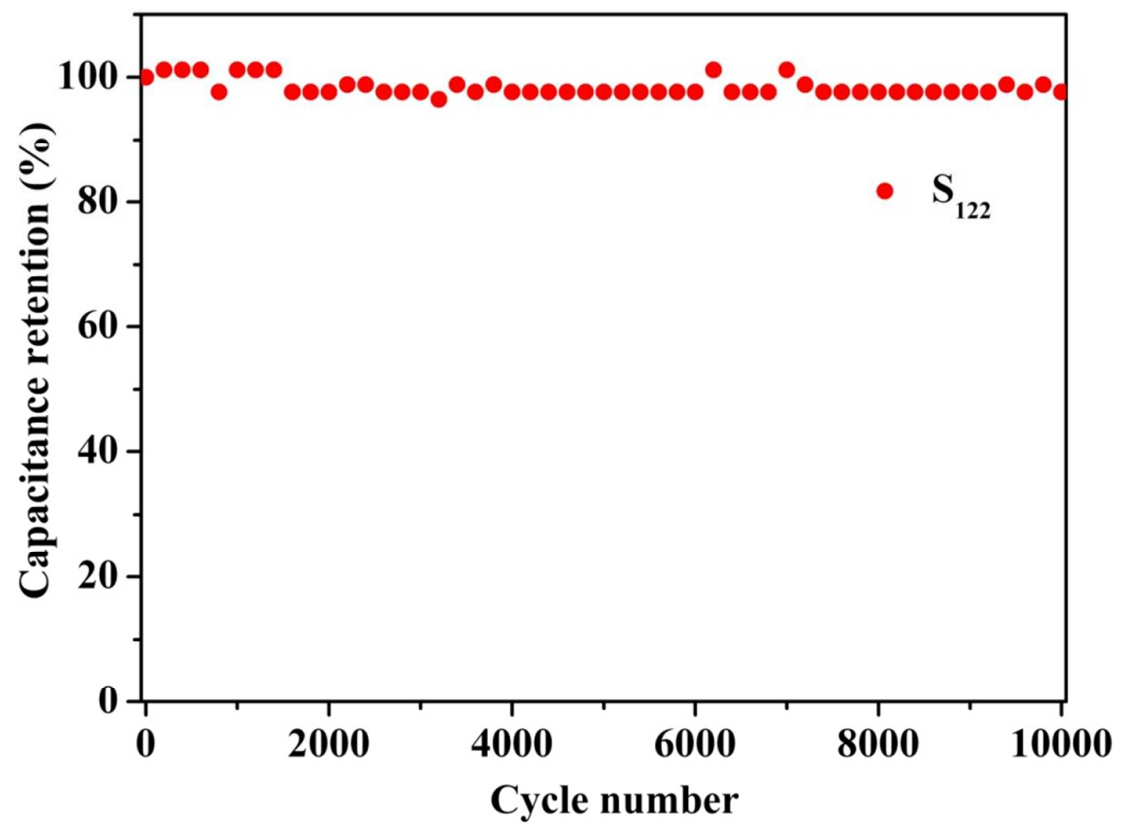

Figure S13. The cycle stability of $S_{122}$ electrode at $5 \mathrm{~A} \mathrm{~g}^{-1}$ in $6.0 \mathrm{M} \mathrm{KOH}$ aqueous electrolytes. 


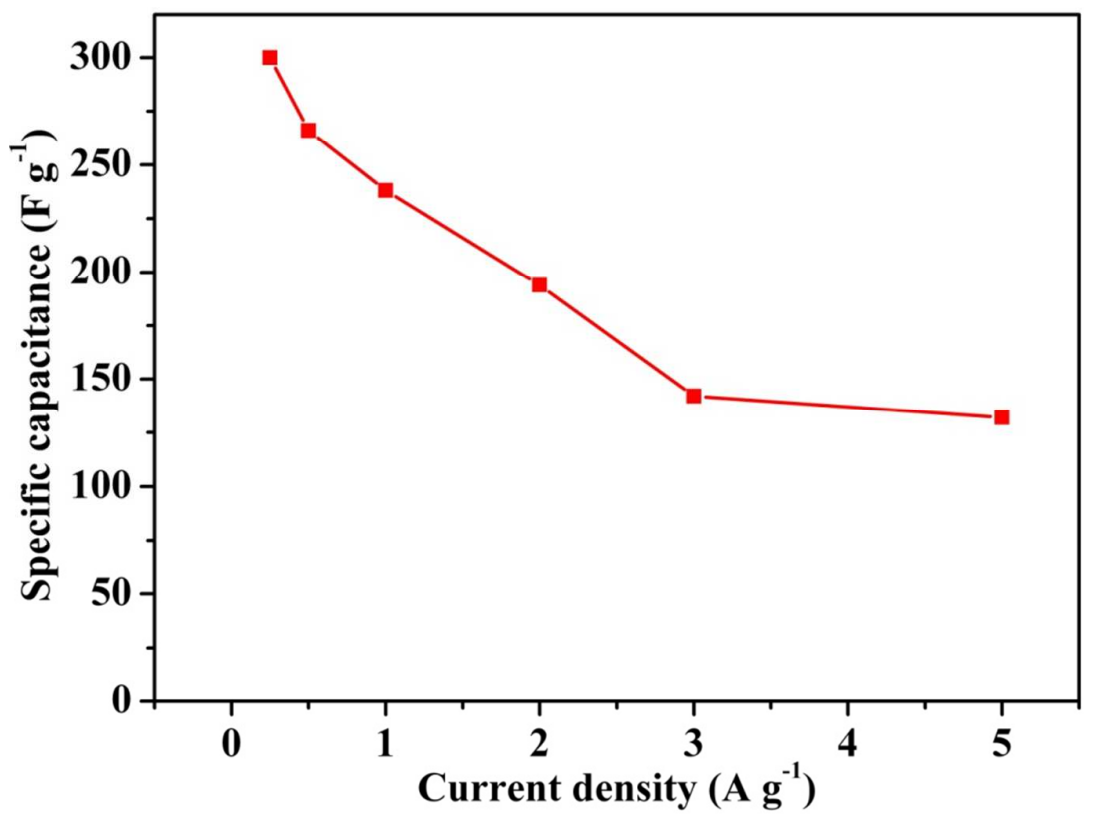

Figure S14. Galvanostatic charge/discharge specific capacitances of the $\mathrm{S}_{122}$ electrode at various current densities in two-electrode system. 
Table S1. The percentage of mesopores of the as-prepared samples.

\begin{tabular}{llllll}
\hline samples & $\begin{array}{l}\mathbf{D} \\
(\mathbf{n m})\end{array}$ & $\begin{array}{l}\mathbf{S}_{\text {BET }} \\
\left(\mathbf{m}^{2} \mathbf{g}^{-\mathbf{1}}\right)\end{array}$ & $\begin{array}{l}\mathbf{V}_{\mathbf{t}} \\
\left(\mathbf{c m}^{\mathbf{3}} \mathbf{g}^{-\mathbf{1}}\right)\end{array}$ & $\begin{array}{l}\mathbf{V}_{\text {mic }} \\
\left(\mathbf{c m}^{3} \mathbf{g}^{-\mathbf{1}}\right)\end{array}$ & $\begin{array}{l}\mathbf{V}_{\text {mes }} / \mathbf{V}_{\mathbf{t}} \\
(\boldsymbol{\%})\end{array}$ \\
\hline $\mathrm{S}_{100}$ & 2.40 & 145.40 & 0.09 & 0.07 & 22.22 \\
$\mathrm{~S}_{102}$ & 2.68 & 760.49 & 0.51 & 0.38 & 25.49 \\
$\mathrm{~S}_{120}$ & 7.31 & 584.98 & 1.07 & 0.19 & 82.24 \\
$\mathrm{~S}_{121}$ & 5.89 & 945.76 & 1.39 & 0.41 & 70.50 \\
$\mathrm{~S}_{122}$ & 3.38 & 805.58 & 0.68 & 0.32 & 52.94 \\
\hline
\end{tabular}

$\mathrm{D}$ : average pore diameter; $\mathrm{S}_{\mathrm{BET}}$ : $\mathrm{BET}$ specific surface area; $\mathrm{V}_{\mathrm{t}}$ : total pore volume; $\mathrm{V}_{\text {mic }}$ : micropore volume and $\mathrm{V}_{\text {mes }}$ : mesopore volume. 
Table S2. Approximate distribution of $\mathrm{N}$ functional groups obtained by fitting the $\mathrm{N}$ 1s core level XPS spectra of the $S_{122}$ and $S_{102}$ samples.

\begin{tabular}{lllll}
\hline \multirow{2}{*}{ Functional groups } & \multicolumn{4}{c}{ \% of total N 1s } \\
\cline { 2 - 5 } & $\mathbf{N}-6$ & $\mathbf{N}-\mathbf{5}$ & $\mathbf{N}-\mathbf{Q}$ & N-X \\
\hline B. E. (eV) & 398.3 & 400.2 & 401.2 & 403.0 \\
$\mathrm{~S}_{102}$ & 25.65 & 48.37 & 5.70 & 20.28 \\
$\mathrm{~S}_{121}$ & 24.76 & 68.76 & 0.01 & 6.47 \\
$\mathrm{~S}_{122}$ & 30.30 & 51.36 & 14.38 & 3.96 \\
\hline
\end{tabular}


Table S3. Comparison of the BET surface area, pore volume and capacitance of different $\mathrm{N}$-doped activated carbons in the literatures.

\begin{tabular}{|c|c|c|c|c|}
\hline N-doped carbons & $\begin{array}{l}\text { BET surface area } \\
\left(\mathbf{m}^{2} \mathbf{g}^{-1}\right)\end{array}$ & $\begin{array}{l}\text { Pore volume } \\
\left(\mathrm{cm}^{3} \mathrm{~g}^{-1}\right)\end{array}$ & $\begin{array}{l}\text { Specific capacitance } \\
\text { (electrolyte) }\end{array}$ & Refs. \\
\hline $\begin{array}{l}\text { Porous N-doped } \\
\text { Nanofibers (N-CNFs) }\end{array}$ & 563 & 0.51 & $\begin{array}{l}202 \mathrm{Fg}^{-1}\left(1 \mathrm{Ag}^{-1}\right) \\
(6 \mathrm{M} \mathrm{KOH})\end{array}$ & {$[\mathrm{S} 1]$} \\
\hline $\begin{array}{l}\text { N-doped graphene fibers } \\
\text { (NG-FMs) }\end{array}$ & 176 & 0.21 & $\begin{array}{l}188 \mathrm{~F} \mathrm{~g}^{-1}\left(5 \mathrm{mv} \mathrm{s}^{-1}\right) \\
(6 \mathrm{M} \mathrm{KOH})\end{array}$ & {$[\mathrm{S} 2]$} \\
\hline $\begin{array}{l}\mathrm{N} \text {-doped graphene nanosheets } \\
\text { (C-NGNSs) }\end{array}$ & 465 & 3.42 & $\begin{array}{l}248 \mathrm{~F} \mathrm{~g}^{-1}\left(5 \mathrm{mv} \mathrm{s}^{-1}\right) \\
(6 \mathrm{M} \mathrm{KOH})\end{array}$ & {$[\mathrm{S} 3]$} \\
\hline $\begin{array}{l}\text { N-doped nanosheets } \\
\text { (N-CNSs) }\end{array}$ & 550 & 3.19 & $\begin{array}{l}249 \mathrm{Fg}^{-1}\left(1 \mathrm{Ag}^{-1}\right) \\
(6 \mathrm{M} \mathrm{KOH})\end{array}$ & [S4] \\
\hline $\begin{array}{l}\text { N-doped networks } \\
\text { (3D N-CNWs) }\end{array}$ & 1442 & 0.85 & $\begin{array}{l}304 \mathrm{Fg}^{-1}\left(0.5 \mathrm{Ag}^{-1}\right) \\
(6 \mathrm{M} \mathrm{KOH})\end{array}$ & [S5] \\
\hline $\begin{array}{l}\text { Micro/mesoporous carbon } \\
\text { (HMCs-800) }\end{array}$ & 1306 & 0.90 & $\begin{array}{l}340 \mathrm{Fg}^{-1}\left(1 \mathrm{Ag}^{-1}\right) \\
(6 \mathrm{M} \mathrm{KOH})\end{array}$ & [S6] \\
\hline $\begin{array}{l}\text { Honeycomb-like porous carbon } \\
\text { (HLPC) }\end{array}$ & 2725 & 1.28 & $\begin{array}{l}342 \mathrm{Fg}^{-1}\left(0.2 \mathrm{Ag}^{-1}\right) \\
(6 \mathrm{M} \mathrm{KOH})\end{array}$ & [S7] \\
\hline $\begin{array}{l}\text { Carbonized eggshell membrane } \\
\text { (CESM-300) }\end{array}$ & 221 & 0.13 & $\begin{array}{l}297 \mathrm{Fg}^{-1}\left(0.2 \mathrm{~A} \mathrm{~g}^{-1}\right) \\
(1 \mathrm{M} \mathrm{KOH})\end{array}$ & [S8] \\
\hline $\mathrm{S}_{102}$ & 760 & 0.51 & $\begin{array}{l}149 \mathrm{Fg}^{-1}\left(1 \mathrm{~A} \mathrm{~g}^{-1}\right) \\
(6 \mathrm{M} \mathrm{KOH})\end{array}$ & This work \\
\hline $\mathrm{S}_{121}$ & 946 & 1.39 & $\begin{array}{l}256 \mathrm{Fg}^{-1}\left(1 \mathrm{Ag}^{-1}\right) \\
(6 \mathrm{M} \mathrm{KOH})\end{array}$ & This work \\
\hline $\mathrm{S}_{122}$ & 806 & 0.68 & $\begin{array}{l}323 \mathrm{Fg}^{-1}\left(1 \mathrm{Ag}^{-1}\right) \\
(6 \mathrm{M} \mathrm{KOH})\end{array}$ & This work \\
\hline
\end{tabular}


Table S4. IR drops of the $S_{121}$ and $S_{122}$ at various current densities.

\begin{tabular}{ccc}
\hline Current density $\left(\mathbf{A ~ g}^{-\mathbf{1}}\right)$ & IR drop of the $\mathbf{S}_{\mathbf{1 2 1}}(\mathbf{V})$ & IR drop of the $\mathbf{S}_{\mathbf{1 2 1}}(\mathbf{V})$ \\
\hline 1 & 0.00034 & 0.00043 \\
5 & 0.00011 & 0.00292 \\
10 & 0.00288 & 0.00400 \\
50 & 0.01552 & 0.02726 \\
100 & 0.04723 & 0.04655 \\
\hline
\end{tabular}


Table S5. The EA results, specific surface area and specific capacitance of the as-prepared samples obtained from different temperatures $(700,800$ and $\left.900{ }^{\circ} \mathrm{C}\right)$.

\begin{tabular}{|c|c|c|c|c|c|c|c|c|}
\hline \multirow{2}{*}{ Samples } & \multicolumn{3}{|c|}{ Elemental analysis } & \multirow{2}{*}{$\begin{array}{c}\mathrm{S}_{\mathrm{BET}} \\
\left(\mathrm{m}^{2} \mathrm{~g}^{-1}\right)\end{array}$} & \multirow{2}{*}{$\begin{array}{c}\mathbf{S}_{\text {mic }} \\
\left(\mathbf{m}^{2} \mathbf{g}^{-1}\right)\end{array}$} & \multirow{2}{*}{$\begin{array}{c}V_{\text {total }} \\
\left(\mathrm{cm}^{3} \mathrm{~g}^{-1}\right)\end{array}$} & \multirow{2}{*}{$\begin{array}{c}D \\
(\mathbf{n m})\end{array}$} & \multirow{2}{*}{$\begin{array}{l}\text { Specific } \\
\text { capacitance } \\
\left(\mathrm{F} \mathrm{g}^{-1}\right)\end{array}$} \\
\hline & $\mathrm{C} \%$ & N\% & $\mathbf{H \%}$ & & & & & \\
\hline$S_{122}-700$ & 59.17 & 9.46 & 2.94 & 718.57 & 646.78 & 0.64 & 3.57 & 185 \\
\hline $\mathrm{S}_{122}-800$ & 69.20 & 8.92 & 1.30 & 805.58 & 632.54 & 0.68 & 3.38 & 323 \\
\hline$S_{122}-900$ & 69.73 & 2.66 & 2.49 & 1187.35 & 1001.08 & 1.07 & 3.62 & 110 \\
\hline
\end{tabular}

$\mathrm{S}_{\mathrm{BET}}$, BET specific surface area;

Smic, micropore specific surface area;

$\mathrm{V}_{\mathrm{t}}$, total pore volume;

$\mathrm{D}$, average pore diameter. 


\section{References}

[S1] L. Chen, X. Zhang, H. Liang, M. Kong, Q. Guan, P. Chen, et al., Synthesis of nitrogen-doped porous carbon nanofibers as an efficient electrode material for supercapacitors. ACS Nano, 2012;6:7092-7102.

[S2] Y. Chang, G. Han, D. Fu, F. Liu, M. Li, Y. Li, Larger-scale fabrication of N-doped graphene-fiber mats used in high-performance energy storage. J Power Sources, 2014;252:113-121.

[S3] Z. Wen, X. Wang, S. Mao, Z. Bo, H. Kim, S. Cui, et al., Crumpled nitrogen-doped graphene nanosheets with ultrahigh pore volume for high-performance supercapacitor. Adv Mater, 2012;24:5610-5616.

[S4] H. Peng, G.F. Ma, K.J. Sun, J.J. Mu, Z.Q. Lei, One-step preparation of ultrathin nitrogen-doped carbon nanosheets with ultrahigh pore volume for high-performance supercapacitors. J Mater Chem A, 2014;2:17297-17301.

[S5] H. Peng, G.F. Ma, K.J. Sun, J.J. Mu, Z. Zhang, Z.Q. Lei, Facile synthesis of poly(p-phenylenediamine)-derived three-dimensional porous nitrogen-doped carbon networks for high performance supercapacitors. J Phys Chem C, 2014;118:29507-29516.

[S6] W.J. Qian, F.X. Sun, Y.H. Xu, L.H. Qiu, C.H. Liu, S.D. Wang, et al., Human hair-derived carbon flakes for electrochemical supercapacitors. Energy Environ Sci, 2014;7:379-386.

[S7] Q.H. Liang, L. Ye, Z.H. Huang, Q. Xu, Y. Bai, F.Y. Kang, et al., A honeycomb-like porous carbon derived from pomelo peel for use in high-performance supercapacitors. Nanoscale, 2014;6:13831-13837.

[S8] Z. Li, L. Zhang, B.S. Amirkhiz, X.H. Tan, Z.W. Xu, H.L. Wang, et al., Carbonized chicken eggshell membranes with 3D architectures as high-performance electrode materials for supercapacitors. Adv Energy Mater, 2012;2:431-437. 